\title{
Novel fusion rules for transform-domain image fusion methods
}

\author{
Chunyu Wei ${ }^{1, a}$, Bingyin Zhou ${ }^{1, b^{*}}$ and Wei Guo ${ }^{1, c}$ \\ ${ }^{1}$ College of Mathematics and Information Sciences, Hebei Normal University, Shijiazhuang, Hebei \\ 050024, P.R. China. \\ achunyuwei@yeah.net, ${ }^{\mathrm{b}}$ zhoubingyin@163.com, ${ }^{\mathrm{c}}$ guowei@chmiot.net
}

Keywords: image fusion, fusion rule, contourlet, shearlet

Abstract. Image fusion has become a major topic in image processing. In transform domain methods, the fusion rules decide the quality of fused image and hence play an important role. Therefore, the key issue is the design of fusion rules which contain low frequency fusion rules and high frequency fusion rules. In this paper, we propose a weighted energy fusion rule for low frequency fusion, and a weighted pixel and regional energy fusion rule for high frequency fusion. Experimental results demonstrate that our fusion rules outperform other fusion rules in most cases.

\section{Introduction}

Image fusion is an important research topic of digital image processing. There are many kinds of fusion applications, including multi-focus image fusion, medical image fusion, infrared and visible image fusion, remote sensing image fusion and so on. The goal of image fusion is to integrate relevant information from different images to construct a new image. The fused image provides better information, and is conducive to the subsequent image processing.

The literature on image fusion is rich and diversified. There are three leading classes of image fusion methods: pixel-level, feature-level, and decision-level [1]. Feature-level and decision-level methods are very much image and application depended. Conversely, pixel-level methods are more popular and can be further classified into two categories: spatial domain fusion, and transform domain fusion. Spatial domain methods perform fusion operations directly on image pixels or regions. They usually operate simplicity and have low computational cost, but also have many shortcomings, such as blocking effect and contrast reduction. Transform domain methods firstly transform the source images into a transform domain to obtain the transform coefficients. Then, the transform coefficients are fused by using some fusion rules. Finally, the fused image is reconstructed by performing the inverse transform. Many kinds of transforms have been applied to image fusion, from wavelet transform to multi-scale geometric analysis tools such as contourlet transform [2,3], shearlet transform [4,5], nonsubsampled contourlet transform (NSCT) [6,7], nonsubsampled shearlet transform (NSST) [8,9], and the combination of NSCT and NSST (NSCT+NSST) [10].

In transform domain methods, the fusion rules decide the quality of fused image and hence play an important role. The fusion rules contain low frequency rules and high frequency rules. The commonly used low frequency fusion rules include averaging fusion rule (AFR) [4,10] and maximum selection fusion rule (MSFR) [11,12]. The AFR makes the fusion results very stable, but would reduce the contrast and yield blur. The MSFR gives prominence to the salient features of original images, which is sensitive to noise and hence may reduce the quality. The single pixel based fusion rules, such as AFR and MSFR, can also be used as high frequency fusion rules. But they are very sensitive to noise so that the noise will be easily introduced to fused image. To reduce the influence of noise, regional based fusion rules, such as regional energy fusion rule (REFR) [3,10] and regional variance fusion rule [4], are commonly used for high frequency fusion.

In this paper, we present a weighted energy fusion rule (WEFR) for low frequency fusion, and a weighted pixel and regional energy fusion rule (WPREFR) for high frequency fusion. The proposed rules are mainly based on the considering of two key points in applications: (1) noise sensitive is more critical for high frequency coefficients, since the noise of images is suppressed in the low frequency 
coefficients; (2) flexible of fusion rules is very important. Experimental results show that our fusion rules improve the performance.

\section{Transform domain fusion rules}

The fusion rule plays an important role in transform domain image fusion methods, since it decides the quality of fused image. The fusion rules contain low frequency rules and high frequency rules.

Low frequency fusion rules. The commonly used low frequency fusion rules are AFR [4,10] and MSFR [11,12]. Let $L_{A}(i, j)$ and $L_{B}(i, j)$ be the low frequency coefficients of source images $f^{A}$ and $f^{B}, \omega_{A}$ and $\omega_{B}$ be the weights, the fused low frequency coefficients $L_{F}(i, j)$ are computed by

$$
L_{F}(i, j)=\omega_{A} L_{A}(i, j)+\omega_{B} L_{B}(i, j) .
$$

The AFR is taking the average of low frequency coefficients as the fused low frequency fusion coefficients, the weights are given by

$$
\omega_{A}=\omega_{B}=\frac{1}{2} .
$$

This makes the fusion results very stable, but would reduce the contrast and yield blur. The MSFR gives prominence to the salient features of the original images, the weights are given by

$$
\omega_{A}=\left\{\begin{array}{ll}
1, & L_{A}(i, j) \geq L_{B}(i, j) \\
0, & L_{A}(i, j)<L_{B}(i, j)
\end{array}, \omega_{B}=1-\omega_{A} .\right.
$$

It is sensitive to noise, and will reduce the quality of fused image.

Here, we propose a WEFR for low frequency fusion, the weights are given by

$$
\omega_{A}=\frac{L_{A}^{2}(i, j)}{L_{A}^{2}(i, j)+L_{B}^{2}(i, j)}, \omega_{B}=1-\omega_{A} .
$$

The values of weights is not just 0,1 , and $1 / 2$, which is more flexible than the above two methods.

High frequency fusion rules. There are two classes of high frequency fusion rules: single pixel based fusion rules and regional based fusion rules. The single pixel based fusion rules, such as AFR and MSFR, can also be used as high frequency rules. However, they are very sensitive to noise, which is more critical for high frequency coefficients. This leads to that the noise will be easily mistaken as useful information and then introduced to the fused image. To reduce the influence of noise, regional based fusion rules such as REFR are commonly used.

The regional energy is defined as

$$
R E(i, j)=\sum_{m=-R}^{R} \sum_{n=-R}^{R} H^{2}(i+m, j+n)
$$

in which $H(i, j)$ is the high frequency coefficient, and $R$ is the radius of the region and we set $R=3$ in our experiments.

Let $H_{A}(i, j)$ and $H_{B}(i, j)$ be the high frequency coefficients of source images $f^{A}$ and $f^{B}$. The fused high frequency coefficients $H_{F}(i, j)$ are computed by

$$
H_{F}(i, j)=\eta_{A} H_{A}(i, j)+\eta_{B} H_{B}(i, j),
$$

where $\eta_{A}$ and $\eta_{B}$ are the weights. For REFR, the weights are given by 


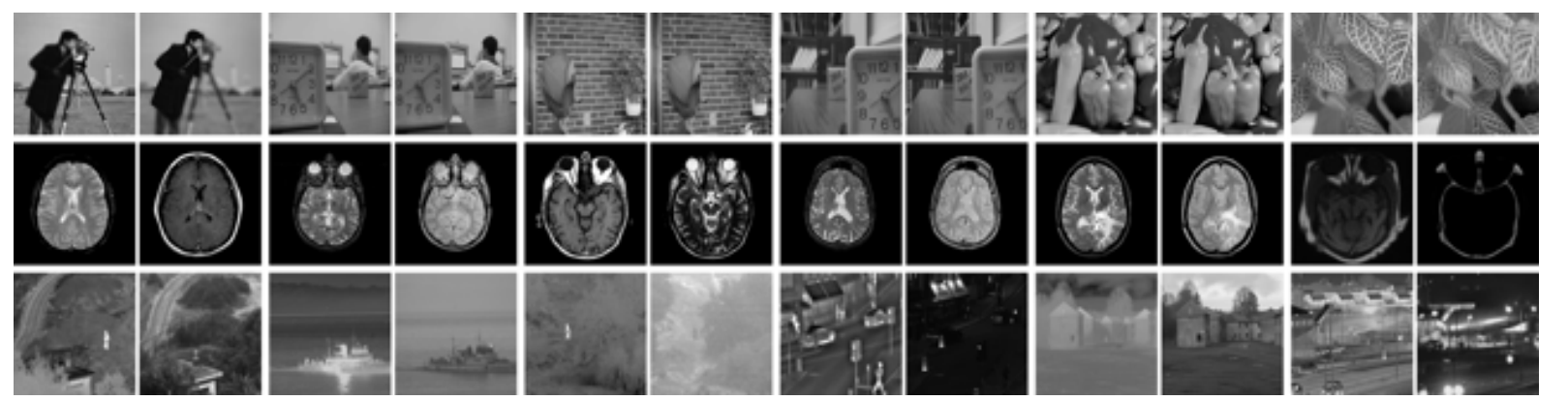

Fig. 1. The 18 pairs of source images: the first row is multi-focus images, the second row is medical images, and the last row is infrared and visible images.

$$
\eta_{A}=\left\{\begin{array}{ll}
1, & R E_{A}(i, j) \geq R E_{B}(i, j) \\
0, & R E_{A}(i, j)<R E_{B}(i, j)
\end{array}, \eta_{B}=1-\eta_{A} .\right.
$$

By individually weighting the regional energy and high frequency coefficient, we propose a WPREFR as the high frequency fusion rule, the weights are given by

$$
\eta_{A}(x)=\left\{\begin{array}{l}
\frac{H_{A}^{2}(x)}{H_{A}^{2}(x)+H_{B}^{2}(x)}, R E_{A}(x) \geq R E_{B}(x) \text { and } H_{\mathrm{A}}(x)<H_{\mathrm{B}}(x) \\
\frac{R E_{A}(x)}{R E_{A}(x)+R E_{B}(x)}, R E_{A}(x) \geq R E_{B}(x) \text { and } H_{\mathrm{A}}(x) \geq H_{\mathrm{B}}(x) \\
\frac{H_{B}^{2}(x)}{H_{A}^{2}(x)+H_{B}^{2}(x)}, R E_{A}(x)<R E_{B}(x) \text { and } H_{\mathrm{A}}(x)>H_{\mathrm{B}}(x) \\
\frac{R E_{B}(x)}{R E_{A}(x)+R E_{B}(x)}, R E_{A}(x)<R E_{B}(x) \text { and } H_{\mathrm{A}}(x) \leq H_{\mathrm{B}}(x)
\end{array}\right.
$$

This method will take the regional energy of pixel $x$ to compute the weight while the regional energy and high frequency coefficient of source image $f^{A}$ are bigger or smaller than the values of source image $f^{B}$ at the same time. Otherwise, it will use the WEFR. The proposed WPREFR can reduce the influence of noise, and also consider the local salience.

\section{Experimental Results and Discussion}

In this section, 18 pairs of $256 \times 256$ images ( 6 pairs of multi-focus images, 6 pairs of medical images, and 6 pairs of infrared and visible images), shown in Fig. 1, are used to evaluate our fusion rules. In the experiments, 5 transform methods are used: contourlet transform, shearlet transform, NSCT, NSST, and NSCT+NSST. Three quality evaluation metrics, $\mathrm{Q}^{\mathrm{AB} / \mathrm{F}}, \mathrm{Q}_{\mathrm{w}}$, and $\mathrm{Q}_{\mathrm{e}}[13]$, are used to evaluate the quality of fused images. Experiments are performed with MATLAB R2009b (32bit) on Q8200 $2.33 \mathrm{GHz}$ and $2.34 \mathrm{GHz}$, Intel(R) Core ${ }^{\mathrm{TM}} 2$ Quad CPU, 3GB of RAM laptop. The toolboxes of contourlet [14], shearlet [15], NSCT [16], and NSST [17] are used for experiments.

The experiments compare different combinations of low frequency fusion rules and high frequency fusion rules mentioned in the previous section. These rules are respectively adopted to fuse transform coefficients by using the selected 5 transform methods. Table 1 shows the comparison results of different fusion rules. The values are averaging for 90 different cases, which are the all combinations of 18 source image pairs and 5 transform methods. It is clear that the proposed fusion rules usually achieve better results. We can observe that the combination of our WEFR and REFR gets the best result for $\mathrm{Q}^{\mathrm{AB} / \mathrm{F}}$ and $\mathrm{Q}_{\mathrm{e}}$, and the combination of our WEFR and our WPREFR gets the best result for $\mathrm{Q}_{\mathrm{w}}$. The results illustrates that the proposed fusion rules are more effective for transform domain fusion methods. 
As a visualization example, Fig. 2-4 show, respectively, the fused images of different fusion rules for three pairs of images. The transform method used is NSCT+NSST. We can observe that the proposed fusion rules usually get better results, not only on the evaluation measurements, but also on the visual quality.

Table 1. The comparison results of different fusion rules. The best results are in italic.

\begin{tabular}{llllll}
\hline Low frequency & High frequency & $\mathrm{Q}^{\mathrm{AB} / F}$ & $\mathrm{Q}_{\mathrm{w}}$ & $\mathrm{Q}_{\mathrm{e}}$ & Time $[\mathrm{s}]$ \\
\hline AFR & REFR & 0.6332 & 0.8258 & 0.4118 & 30 \\
AFR & Our WPREFR & 0.6282 & 0.8324 & 0.3989 & 40 \\
MSFR & REFR & 0.6379 & 0.8456 & 0.4188 & 30 \\
MSFR & Our WPREFR & 0.6313 & 0.8509 & 0.4041 & 39 \\
Our WEFR & REFR & 0.6392 & 0.8494 & 0.4207 & 30 \\
Our WEFR & Our WPREFR & 0.6330 & 0.8550 & 0.4052 & 40 \\
\hline
\end{tabular}
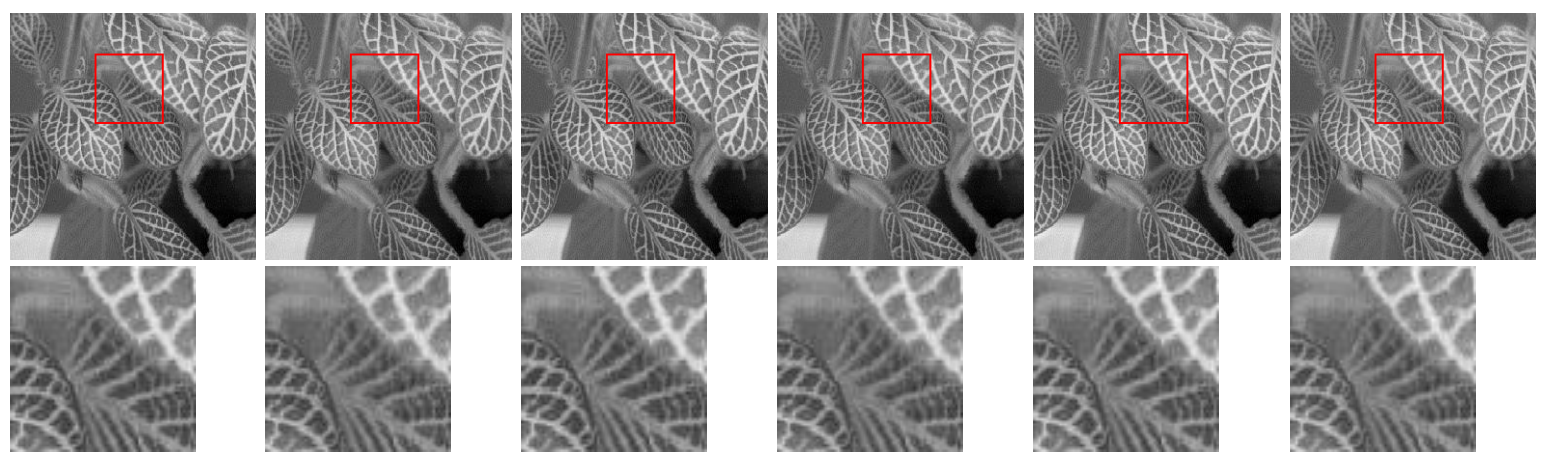

Fig. 2. The fused images of a multi-focus image pair: the $i$-th column is obtained by using the combination of low and high frequency rules shown in the $i$-th row of Table 1.
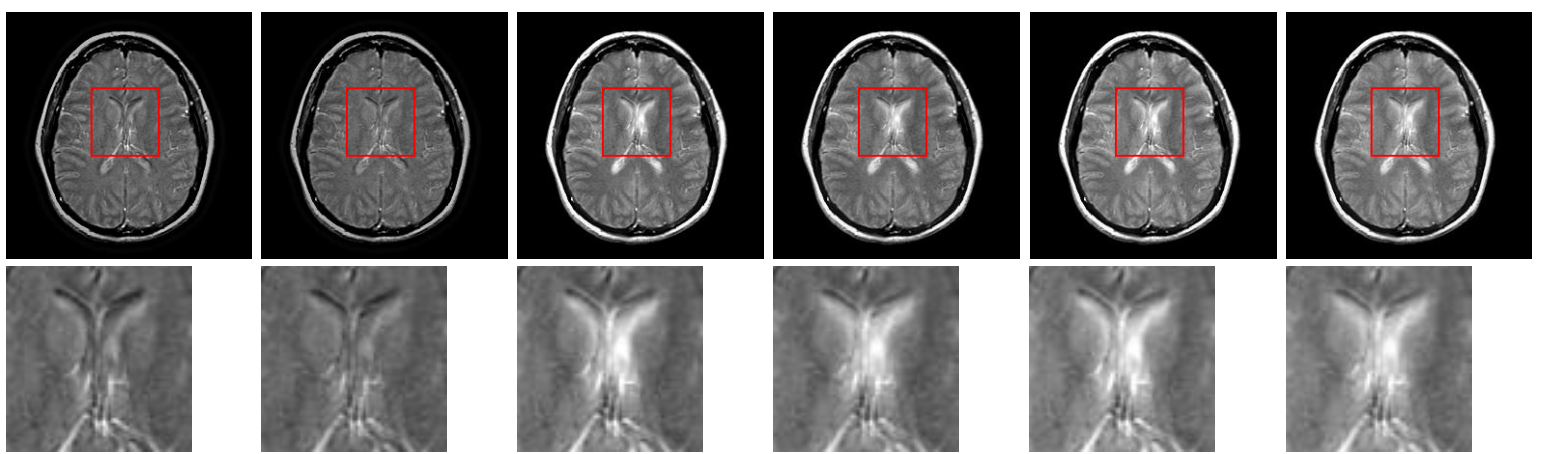

Fig. 3. The fused images of a medical image pair: the $i$-th column is obtained by using the combination of low and high frequency rules shown in the $i$-th row of Table 1.
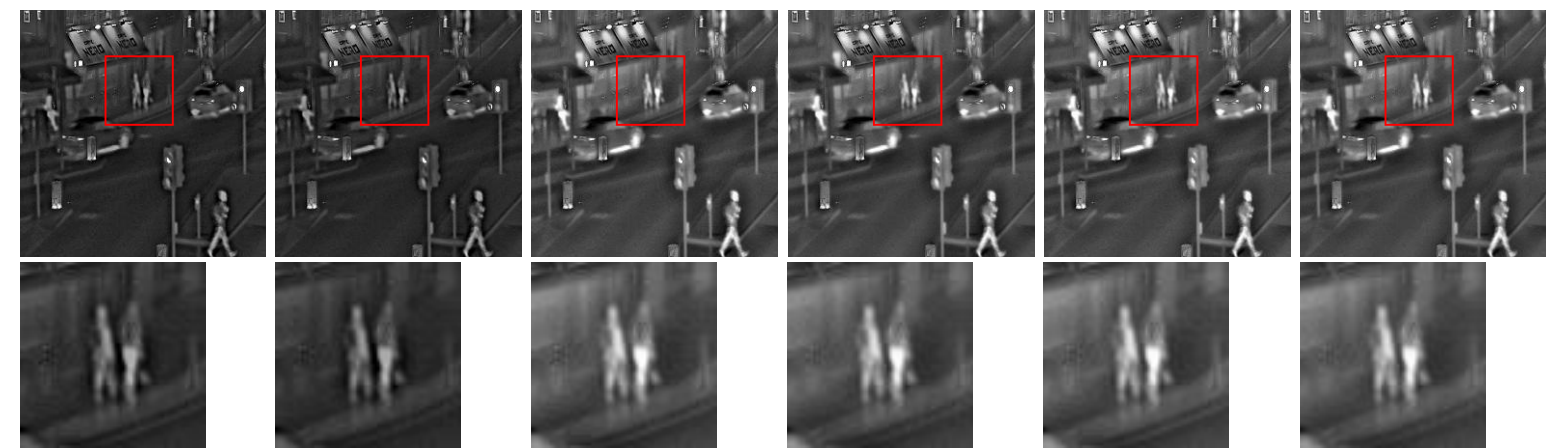

Fig. 4. The fused images of an infrared and visible image pair: the $i$-th column is obtained by using the combination of low and high frequency rules shown in the $i$-th row of Table 1. 


\section{Conclusions}

In this paper, we proposed the WEFR as the low frequency fusion rule, and the WPREFR as the high frequency fusion rule. Experimental results demonstrate that our fusion rules usually outperform other fusion rules.

\section{Acknowledgments}

The authors would like to thank the editor and anonymous reviewers for their detailed review and valuable comments. This work was supported by the NSF of China (No. 11301137), the NSF of Hebei Province, China (No. A2014205100), the Educational Commission of Hebei Province, China (No. ZD2014062), and the Doctoral Fund of Hebei Normal University, China (No. L2012B02).

\section{References}

[1] S. Li, B. Yang, J. Hu, Information Fusion 12 (2) (2011) 74-84.

[2] L. Yang, B. L. Guo, W. Ni, Neurocomputing 72 (1-3) (2008) 203-211.

[3] C. Li, X. Yang, B. Chu, W. Lu, L. Pang, in: Proceedings of the 3rd IEEE International Conference on Computer Science and Information Technology, Vol. 5, IEEE, Chengdu, China, 2010, pp. 246-249.

[4] Q.Miao, C. Shi, P. Xu, M. Yang, Y. Shi, Chinese Optics Letters 9 (4) (2011) 1-5.

[5] X. Liu, Y. Zhou, J. Wang, AEU - International Journal of Electronics and Communications 68 (6) (2014) 471-477.

[6] Q. Zhang, B. L. Guo, Signal Processing 89 (7) (2009) 1334-1346.

[7] Y. Yang, S. Tong, S. Huang, P. Lin, IEEE Sensors Journal 15 (5) (2015) 2824-2838.

[8] Y. Cao, S. Li, J. Hu, in: Proceedings of the 6th International Conference on Image and Graphics, IEEE, Hefei, China, 2011, pp. 17-21.

[9] G. Gao, L. Xu, D. Feng, IET Image Processing 7 (6) (2013) 633-639.

[10] A.-U. Moonon, J. Hu, Sensing and Imaging 16 (1) (2015) 1-16.

[11] Y. Liu, S. Liu, Z. Wang, Information Fusion 24 (2015) 147-164.

[12] T. Xiang, L. Yan, R. Gao, Infrared Physics \& Technology 69 (2015) 53-61.

[13] G. Piella, H. Heijmans, in: Proceedings of 2003 International Conference on Image Processing, IEEE , Barcelona, Spain, 2003, pp. 173-176.

[14] M. N. Do, M. Vetterli, in: Proceedings of 2002 International Conference on Image Processing, Vol. 1, IEEE, Rochester, NY, USA, 2002, pp. 357-360.

[15] W. Q. Lim, IEEE Transactions on Image Processing 19 (5) (2010) 1166-1180.

[16] A. L. da Cunha, J. Zhou, M. N. Do, IEEE Transactions on Image Processing 15 (10) (2006) 3089-3101.

[17] G. Easley, D. Labate, W.-Q. Lim, Applied and Computational Harmonic Analysis 25 (1) (2008) $25-46$. 